PUPT-1770

NSF-ITP-98-022

hep-th/9803023

\title{
Scalar Absorption and the Breaking of the World Volume Conformal Invariance
}

\author{
Steven S. Gubser ${ }^{a, b}$, Akikazu Hashimoto ${ }^{b}$, Igor R. Klebanov ${ }^{a, b}$, and Michael Krasnitz ${ }^{a}$ \\ ${ }^{a}$ Joseph Henry Laboratories \\ Princeton University \\ Princeton, New Jersey 08544 \\ ${ }^{b}$ Institute for Theoretical Physics \\ University of California \\ Santa Barbara, CA 93106
}

\begin{abstract}
We investigate a version of fixed scalars for non-dilatonic branes which correspond to dilatations of the brane world-volume. We obtain a cross-section whose world-volume interpretation falls out naturally from an investigation of the breaking of conformal invariance by the irrelevant Born-Infeld corrections to Yang-Mills theory. From the same irrelevant world-volume operator we obtain the leading correction to the crosssections of minimal scalars. This correction can be obtained in supergravity via an improved matching of inner and outer solutions to the minimal wave equation.
\end{abstract}

March 1998 


\section{Introduction}

Recently there has been major progress in understanding the strong coupling limit of large $N$ gauge theory $[1,2,3,4,5,6,7,8,9,10,11,12,13]$. Maldacena's work [6] has connected the applications of the large $N$ limit of coincident D3 branes to black 3-branes $[1,2,3,4]$ with Polyakov's sigma model approach to confining strings in large $N$ gauge theories [5]. The conjecture of [6] relates the world volume theories of certain coincident branes to superstring or M-theory on the backgrounds consisting of anti-deSitter $(A d S)$ spaces times spheres, which arise as the throat limits of the relevant black brane geometries [14, 15].

Earlier calculations revealing connections between correlators in the world volume theories and low-energy absorption in the entire brane metric may be found in [2, 3, 4]. Taking the throat limit identifies the "universal" part of the metric which determines the correlation functions in the conformal limit $[6,7,9]$. Indeed, because the isometry groups of $A d S$ spaces are conformal groups, it is sensible for them to be associated with a conformal theory on the world-volume. However, brane geometries are only $A d S$ out to a characteristic radius $R$ which scales as a power of the number of branes. Furthermore, when one specifies the world-volume theory with the DBI action, powers of the string scale $\alpha^{\prime}$ suppress the nonrenormalizable interactions. We find that, even for large $N$ and strong coupling, when the entire brane geometry is smooth [2], these corrections are easily detectable in the energy dependence of the absorption cross-sections. The purpose of this paper is to identify and explain these effects.

To examine the departures from conformal invariance more closely, we propose two concrete probes. On the one hand, we examine the calculations of absorption of minimally coupled scalars [2] in more detail. The wave equation for minimal scalars propagating in the extremal 3-brane background is given by

$$
\left[\rho^{-5} \frac{d}{d \rho} \rho^{5} \frac{d}{d \rho}+1+\frac{(\omega R)^{4}}{\rho^{4}}\right] \phi(\rho)=0 .
$$

The coupling to the background geometry is controlled by dimensionless parameter

$$
(\omega R)^{4} \sim \omega^{4} \kappa N
$$

where the gravitational constant $\kappa \sim g_{Y M}^{2}\left(\alpha^{\prime}\right)^{2}$. In [2] a general relation was proposed between a certain limit of the type IIB supergravity in the self-dual 3-brane background, and the corresponding limit of the world volume gauge theory. This is the double-scaling limit,

$$
g_{Y M}^{2} N \rightarrow \infty, \quad \omega^{2} \alpha^{\prime} \rightarrow 0,
$$

where $\omega^{4}\left(\alpha^{\prime}\right)^{2} g_{Y M}^{2} N \sim(\omega R)^{4}$ is kept fixed and small. One expects the absorption crosssection to contain corrections of higher order in this parameter: ${ }^{1}$

$$
\sigma \sim \kappa^{2} N^{2} \omega^{3}\left(1+c_{1}(\omega R)^{4}+c_{2}(\omega R)^{8}+\ldots\right)
$$

In this paper we present a systematic procedure for obtaining such corrections to the absorption cross-section. Somewhat surprisingly, we find that the leading correction contains

\footnotetext{
${ }^{1} \mathrm{~A}$ similar point was discussed in [16].
} 
a logarithmic term

$$
\sigma \sim \kappa^{2} N^{2} \omega^{3}\left(1+b_{1}^{\prime}(\omega R)^{4} \log (\omega R)+b_{1}(\omega R)^{4}+\ldots\right)
$$

which dominates in the small $\omega R$ limit over the $(\omega R)^{4}$ term. Nevertheless, this expansion is consistent with the double-scaling limit (2). The logarithmic term encodes the leading departure from the conformal limit. For the D3-branes, this breakdown is naturally interpreted in terms of the DBI action: at lowest order, the DBI action reduces to $\mathcal{N}=4$ SYM theory, which is conformal in 4 dimensions; but as one proceeds to higher orders, non-renormalizable interactions enter into the Lagrangian. We argue that the $\operatorname{tr} F^{4}$ term from the DBI action $[17,18,19]$ in fact reproduces the peculiar logarithmic form of the leading correction to the absorption probability.

The other probe is a higher-dimensional analogue of the fixed scalars considered in four and five dimensions $[20,21,22,23,24,25]$. In a compactified geometry where the brane is wrapped around a torus, the scalar we consider is the volume of the torus. Variations of this scalar correspond to dilatations of the world volume; hence it couples to the trace of the stress-energy tensor. This trace vanishes for a conformal theory, and correspondingly the absorption of the fixed scalar is suppressed by $(\omega R)^{4}$ where $\omega$ is the energy. It turns out for the D3-brane that the same $\operatorname{tr} F^{4}$ term in the DBI action gives a contribution to the stress-energy tensor which explains the absorption probability at leading order in low energies.

\section{Fixed Scalars and Conformal Operators}

It is by now well-known that, in the background of certain supersymmetric black holes in 4 and 5 dimensions, there exist non-minimally coupled massless scalar fields. One class of such fields, called the fixed scalars, was discovered in $[26,27,28] .{ }^{2}$ Due to the non-minimal couplings, the low-energy absorption cross-sections for such fields are suppressed compared to those of the minimally coupled scalars [20, 21, 22, 23]. In the effective string models of $D=4$ and $D=5$ black holes this suppression has a natural explanation: while the minimal scalars couple to marginal operators, the non-minimal ones couple to irrelevant operators $[21,22,23]$. Such operators are ignored in the conformal limit, but are well-known to be present in the non-polynomial actions of the DBI type.

In this section we calculate the absorption cross-sections of fixed scalars by charged black holes in $D>5$, and find the suppression at low energies similar to that discovered in $D=4$ and 5. Particularly interesting from our point of view are the cases $D=7,6$ and 9 , which are related to multiply wrapped D3, M5 and M2 branes respectively. These are precisely the theories that have been receiving much attention recently, and the fixed scalars provide a novel way of probing them.

The semiclassical absorption cross-sections of minimally coupled scalars (such as the dilatons) were studied in $[2,3,4]$ and were found to be consistent with world volume considerations. For instance, the leading coupling of the dilaton to the $U(N)$ theory on $N$

\footnotetext{
${ }^{2}$ For $D=5$ there exists yet another class, called the intermediate scalars [29].
} 
coincident D3-branes is

$$
\int d^{4} x \phi(x) \mathcal{O}_{4}
$$

where $\mathcal{O}_{4}$ is the marginal operator which changes $g_{Y M}$,

$$
\mathcal{O}_{4}=\operatorname{tr} F^{2}+\ldots
$$

At low energies the absorption cross-section is found to behave as $\sigma \sim \kappa^{2} N^{2} \omega^{3}$, consistent with the fact that the exact dimension of $\mathcal{O}_{4}$ is equal to 4 . Other fields that act as scalars from the point of view of the $D=7$ black hole include the gravitons polarized in the internal dimensions (along the D3 branes). Their world volume coupling is given by

$$
\int d^{4} x \frac{1}{2} h_{\mu \nu} T^{\mu \nu}
$$

where $T^{\mu \nu}$ is the stress-energy tensor. Now, we may consider a particular scalar field, $h_{\mu}^{\mu}$. Since its vertex operator is $T_{\mu}^{\mu}$, this field obviously decouples in the conformal limit. This is precisely the property expected of a fixed scalar, and we will show that the corresponding linearized equation indeed contains a non-minimal term.

We have concluded that, for D3 branes, $h_{\mu}^{\mu}$ does not couple to a marginal (dimension 4) operator. We may deduce the leading operator which it couples to from the well-known structure of the DBI action $[17,18,19]$. To order $F^{4}$, we have

$$
S_{\mathrm{DBI}}=\frac{1}{4 g_{Y M}^{2}} \int d^{4} x\left[\operatorname{tr} F_{\mu \nu}^{2}-2\left(\pi \alpha^{\prime}\right)^{2} \mathcal{O}_{8}+\ldots\right] .
$$

The operator ${ }^{3}$

$$
\mathcal{O}_{8}=\frac{2}{3} \operatorname{tr}\left(F_{\mu \nu} F_{\rho \nu} F_{\mu \lambda} F_{\rho \lambda}+\frac{1}{2} F_{\mu \nu} F_{\rho \nu} F_{\rho \lambda} F_{\mu \lambda}-\frac{1}{4} F_{\mu \nu} F_{\mu \nu} F_{\rho \lambda} F_{\rho \lambda}-\frac{1}{8} F_{\mu \nu} F_{\rho \lambda} F_{\mu \nu} F_{\rho \lambda}\right)
$$

has bare dimension 8 and obviously breaks conformal invariance. Thus, the trace of the stress-energy tensor calculated from this term is also of dimension 8, i.e. the lowest dimension coupling of the fixed scalar to the world volume is of the form

$$
\int d^{4} x h_{\mu}^{\mu} \mathcal{O}_{8}\left(2 \pi \alpha^{\prime}\right)^{2}
$$

The leading contribution to the 2-point function is a 3-loop diagram, which scales as

$$
\left\langle\mathcal{O}_{8}(x) \mathcal{O}_{8}(0)\right\rangle \sim \frac{N^{2}\left(N g_{Y M}^{2}\right)^{2}}{x^{16}} .
$$

In fact, our absorption calculations will give us reasons to believe that this formula is true non-perturbatively, i.e. this operator does not receive any anomalous dimension in the CFT. Assuming this, performing the Fourier transform and isolating the imaginary part, we find that the absorption cross-section should behave as

$$
\sigma \sim N^{4} \kappa^{4} \omega^{11}
$$

\footnotetext{
${ }^{3}$ We have not exhibited the dependence of this operator on the scalars and the fermions. We believe that these extra terms are determined by supersymmetry.
} 
In the next subsection we will see that precisely this scaling results from the semiclassical gravity calculation.

A parallel analysis may be performed also for the coincident M5 and M2 branes. In both cases we find that trace of the 11 dimensional graviton over indices parallel to the brane is the fixed scalar field, and its leading order coupling is to a relevant operator of twice the marginal dimension. Of course, in these cases comparison with the world volume considerations is weaker since the theory of multiple coincident branes is poorly understood.

\subsection{Semiclassical absorption of fixed scalars}

We obtain a charged black hole in $D=10-p$ by wrapping some number of Dirichlet $p$ branes over $T^{p}$. The part of the $D$-dimensional effective action that will be relevant for our calculations is [30]

$$
\int d^{D} x \sqrt{-g}\left(R-\frac{1}{2} \partial_{m} \lambda \partial^{m} \lambda-e^{\beta \lambda} G_{m n} G^{m n}\right)
$$

where

$$
\beta=\sqrt{\frac{2(D-1)}{D-2}},
$$

and $G_{m n}$ is the $U(1)$ field strength. The fixed scalar $\lambda$ is a certain linear combination of $\log V$ and the 10-dimensional dilaton $\phi$ ( $V$ is the internal volume of the brane measured in the 10-dimensional Einstein metric),

$$
\beta \lambda=\frac{D-7}{2} \phi-\left(\frac{2 D-6}{D-2}\right) \log V .
$$

The static charged black hole solution is

$$
\begin{gathered}
d s^{2}=A^{\frac{1}{D-2}}\left(-A^{-1} d t^{2}+d r^{2}+r^{2} d \Omega_{D-2}^{2}\right), \\
G_{r t}=\frac{1}{\sqrt{2}} \partial_{r} A^{-1}, \quad \lambda=\frac{\beta}{2} \log A, \\
A(r)=1+\frac{R^{D-3}}{r^{D-3}}=1+\frac{2 Q}{(D-3) r^{D-3}} .
\end{gathered}
$$

We will see that perturbing about this solution works similarly to the $D=5$ case which has already been worked out for the triply charged black hole. In fact, setting two of the three charges to zero in [23] we may immediately obtain the fixed scalar fluctuation equation for $D=5$. This is one of the consistency checks on our new calculations. In fact, we will be able to work out the equations for a general $D$. The cases of special interest to us are $\mathrm{D}=7$, corresponding to coincident D3 branes in 10 dimensions; $\mathrm{D}=6$, corresponding to coincident M5 branes in 11 dimensions or D4 brane in 10 dimensions; and $\mathrm{D}=9$, corresponding to coincident M2 branes in 11 dimensions or strings in 10 dimensions.

One may be concerned that the $D=7$ case should be treated separately because the solution also includes the 5 -form background $H=\star G$. Thus, a priori the action is

$$
\int d^{7} x \sqrt{-g}\left(R-\frac{1}{2} \partial_{m} \lambda \partial^{m} \lambda-e^{\beta \lambda} G_{m n} G^{m n}-\frac{2}{5 !} e^{-\beta \lambda} H_{m_{1} \ldots m_{5}} H^{m_{1} \ldots m_{5}}\right) .
$$


However, we may dualize the $H^{2}$ term into the $G^{2}$ term, so that the action is equivalent to

$$
\int d^{7} x \sqrt{-g}\left(R-\frac{1}{2} \partial_{m} \lambda \partial^{m} \lambda-2 e^{\beta \lambda} G_{m n} G^{m n}\right) .
$$

This makes it look essentially the same as the problem in $D \neq 7$. The extra factor of 2 in front of the $G^{2}$ term is compensated by the fact that the classical electric field has an extra $1 / \sqrt{2}$ : in this case

$$
G_{r t}=\frac{1}{2} \partial_{r} A^{-1} .
$$

In studying the propagation of fixed scalars, special care needs to be taken to account for mixing with the gravitational field. This mixing can be traced to the fact that $\lambda$ couples to background electric field of the black hole. Fortunately, the methods for disentangling this mixing have been developed in [23]. Their application here is a straightforward generalization of these methods, and we just summarize the results.

The general formula for the fixed scalar potential is

$$
\frac{2(D-1)^{2}(D-3)^{2} Q^{2}}{r^{2}\left[(D-1) Q+(D-2)(D-3) r^{D-3}\right]^{2}} \text {. }
$$

Using $R^{D-3}=\frac{2 Q}{D-3}$, we find that the fixed scalar fluctuations in $\mathrm{D}$ dimensions obey the equation

$$
\left[r^{-(D-2)} \partial_{r} r^{D-2} \partial_{r}+\omega^{2}\left(1+\frac{R^{D-3}}{r^{D-3}}\right)-\frac{2(D-1)^{2}(D-3)^{2} R^{2(D-3)}}{r^{2}\left[(D-1) R^{D-3}+2(D-2) r^{D-3}\right]^{2}}\right] \lambda=0 .
$$

It remains to find an approximate solution of this equation for low energies and derive the absorption cross-section. As in previous work [20, 21, 22, 23], we divide space into three regions and match. In the near region the equation is

$$
\left[\rho^{-(D-2)} d_{\rho} \rho^{D-2} d_{\rho}+\frac{(\omega R)^{D-3}}{\rho^{D-3}}-\frac{2(D-3)^{2}}{\rho^{2}}\right] \lambda_{I}=0 .
$$

where $\rho=\omega r$. Letting

$$
\rho=A z^{2 /(5-D)}, \quad A^{D-5}=\frac{4(\omega R)^{D-3}}{(D-5)^{2}}
$$

we find

$$
\lambda_{I}=z^{(D-3) /(D-5)} H_{\nu}(z)
$$

where

$$
\nu=\frac{3(D-3)}{D-5} .
$$

In the intermediate region the $\omega$ term is irrelevant and we get the solution

$$
\lambda_{I I}(r)=\frac{B r^{D-3}}{(D-1) R^{D-3}+2(D-2) r^{D-3}} .
$$


Matching with the near region, we obtain

$$
B=\left[\frac{4}{(D-5)^{2}}\right]^{(3-D) /(D-5)} \frac{(D-1) \Gamma(\nu) 2^{\nu}}{\pi}(\omega R)^{-2(D-3) /(D-5)} .
$$

In the far region we have the equation

$$
\left[r^{-(D-2)} \partial_{r} r^{D-2} \partial_{r}+\omega^{2}\right] \lambda_{I I I}=0
$$

Its solution is

$$
\lambda_{I I I}=C \rho^{-\mu} J_{\mu}(\rho),
$$

where $\mu=(D-3) / 2$. Matching to the intermediate region, we get

$$
C=\frac{2^{\mu} \Gamma(\mu+1)}{2(D-2)} B \text {. }
$$

The invariant flux is given by $(1 / 2 i)\left(\lambda^{*} \partial_{r} r^{D-2} \lambda-\right.$ c.c. $)$. Taking the ratio of the flux at the horizon to the the incoming part of the flux at infinity, we get the absorption probability

$$
P=\frac{4}{|C|^{2}} \frac{(D-5)}{2} A^{D-3}
$$

which translates into

$$
P=(D-5)\left[\frac{4}{(D-5)^{2}}\right]^{3(D-3) /(D-5)} \frac{(D-2)^{2}}{(D-1)^{2}} \frac{8 \pi^{2}}{2^{2(\mu+\nu)}(\Gamma(\nu))^{2}(\Gamma(\mu+1))^{2}}(\omega R)^{\frac{(D-3)(D+1)}{D-5}} .
$$

The s-wave absorption cross-section is given by

$$
\sigma=\frac{(2 \sqrt{\pi})^{D-3} \Gamma\left(\frac{D-1}{2}\right)}{\omega^{D-2}} P
$$

Thus, we find

$$
\sigma=(D-5)\left[\frac{4}{(D-5)^{2}}\right]^{3(D-3) /(D-5)} \frac{(D-2)^{2}}{(D-1)^{2}} \frac{8 \pi^{2}(2 \sqrt{\pi})^{D-3}}{2^{2(\mu+\nu)}(\Gamma(\nu))^{2} \Gamma(\mu+1)} R^{\frac{(D-3)(D+1)}{D-5}} \omega^{\frac{5 D-13}{D-5}} .
$$

Let us exhibit the scaling of the cross-section with the number of branes and the energy:

$$
\sigma \sim N^{\frac{D+1}{D-5}} \omega^{\frac{5 D-13}{D-5}}
$$

For $N$ coincident D3 branes, which correspond to the $D=7$ black hole, we find

$$
\sigma_{D 3} \sim \kappa_{10}^{4} N^{4} \omega^{11}
$$

As explained in the preceding section, this scaling shows that the exact dimension of the operator $\mathcal{O}_{8}$, which the fixed scalar couples to, is equal to 8. Thus, its anomalous dimension vanishes. We have, therefore, found another situation where gravity gives us a "proof" of a non-renormalization theorem for an operator in the world volume theory. We believe that in 
the gauge theory this theorem follows from the existence of the supersymmetric DBI action, and from the fact that insertions of $\mathcal{O}_{8}$ can be obtained by differentiating the path integral with respect to $\alpha^{\prime}$.

While our route towards the operator $\mathcal{O}_{8}$ involved using the DBI action, which breaks the conformal invariance, the operator itself is expected to be one of the chiral operators of the $\mathcal{N}=4$ SYM theory. ${ }^{4}$ This is required by the statement that the chiral operators are in one to one correspondence with the massless modes of type IIB supergravity [7, 8, 9]. Chiral operators involving tr $F^{4}$ have indeed been found in [31]. From the form of the fixed scalar equation in the throat region, (17), we find that the AdS mass-squared of the corresponding state is

$$
m^{2}=32 / R^{2}
$$

Thus, we believe that $\mathcal{O}_{8}$ should be identified with the $k=0$ (the $S O(6)$ singlet) state in the tower

$$
m^{2}=(k+4)(k+8) / R^{2},
$$

which appears in type IIB supergravity on $A d S_{5} \times S^{5}$ [32].

For $N$ coincident M5 branes, which correspond to the $D=6$ black hole, we find

$$
\sigma_{M 5} \sim \kappa_{11}^{14 / 3} N^{7} \omega^{17}
$$

This indicates that the fixed scalar couples to an operator of dimension 12 on the 6dimensional world volume.

For $N$ coincident M2 branes, which correspond to the $D=9$ black hole, we find

$$
\sigma_{M 2} \sim \kappa_{11}^{10 / 3} N^{5 / 2} \omega^{8}
$$

This indicates that the fixed scalar couples to an operator of dimension 6 on the 3-dimensional world volume. It is also known that in the effective string models describing black holes in $D=4$ and 5 , the fixed scalars couple to dimension 4 operators [21, 22, 23]. Thus, in all known cases we find that the leading relevant term in the non-linear action, which couples to the fixed scalar, has twice the marginal dimension.

\section{$3 \quad$ Higher order corrections to minimal scalar coupling}

In this section we will exhibit a technique for obtaining systematic corrections to the minimal scalar cross-section. We will start with the D3-brane in section 3.1, then proceed to the M5brane in section 3.2. We will find it useful that the minimal scalar equation in the 3-brane background has an inversion symmetry which interchanges the throat and the asymptotic regions. There is also a similar symmetry which interchanges the throat of the M2-brane with the asymptotic region of the M5-brane and vice versa. This guarantees, among other things, that the absorption probabilities for the M2 and M5 cases are identical.

Let us start with exhibiting this inversion symmetry for the extremal 3-brane. The minimal massless scalar equation is given in (1). If we make a substitution, $\phi(\rho)=\rho^{-2} \chi(\rho)$,

\footnotetext{
${ }^{4}$ We thank O. Aharony, H. Ooguri and J. Maldacena for emphasizing this to us.
} 
then

$$
\left[\frac{d^{2}}{d \rho^{2}}+\frac{1}{\rho} \frac{d}{d \rho}+1-\frac{4}{\rho^{2}}+\frac{(\omega R)^{4}}{\rho^{4}}\right] \chi=0 .
$$

On the other hand, if we introduce a variable $y=(\omega R)^{2} / \rho$, then

$$
\left[\frac{d^{2}}{d y^{2}}+\frac{1}{y} \frac{d}{d y}+1-\frac{4}{y^{2}}+\frac{(\omega R)^{4}}{y^{4}}\right] \chi=0,
$$

which is the same equation! So, there is a inversion symmetry that interchanges the $\operatorname{AdS}$ and the asymptotic regions.

This symmetry is even evident in the metric. If we start with the extremal metric

$$
\begin{gathered}
d s^{2}=A(r)^{-1 / 2} d x_{\|}^{2}+A(r)^{1 / 2}\left(d r^{2}+r^{2} d \Omega_{5}^{2}\right), \\
A(r)=1+\frac{R^{4}}{r^{4}},
\end{gathered}
$$

and define a new coordinate, $z=R^{2} / r$, then the metric becomes

$$
d s^{2}=\frac{R^{2}}{z^{2}}\left[A(z)^{-1 / 2} d x_{\|}^{2}+A(z)^{1 / 2}\left(d z^{2}+z^{2} d \Omega_{5}^{2}\right)\right] .
$$

So, after a conformal transformation the metric looks the same in terms of $z$.

Now consider the massless equation in the background of a M5-brane,

$$
\left[\rho^{-4} \frac{d}{d \rho} \rho^{4} \frac{d}{d \rho}+1+\frac{(\omega R)^{3}}{\rho^{3}}\right] \phi(\rho)=0 .
$$

If we make a substitution, $\phi(\rho)=\rho^{-3 / 2} \psi(\rho)$, then

$$
\left[\frac{d^{2}}{d \rho^{2}}+\frac{1}{\rho} \frac{d}{d \rho}+1-\frac{9}{4 \rho^{2}}+\frac{(\omega R)^{3}}{\rho^{3}}\right] \psi(\rho)=0 .
$$

Here the absorption probability is known to be [2]

$$
P=\frac{\pi}{9}(\omega R)^{9} \text {. }
$$

For the M2-brane the equation is

$$
\left[\rho^{-7} \frac{d}{d \rho} \rho^{7} \frac{d}{d \rho}+1+\frac{(\omega R)^{6}}{\rho^{6}}\right] \phi(\rho)=0 .
$$

Using the variable $y=(\omega R)^{3} /\left(2 \rho^{2}\right)$, and setting $\phi=y^{3 / 2} \chi$, one gets

$$
\left[\frac{d^{2}}{d y^{2}}+\frac{1}{y} \frac{d}{d y}+1-\frac{9}{4 y^{2}}+\frac{(\omega R)^{3}}{8 y^{3}}\right] \chi(y)=0,
$$

which is the same equation as for the M5-brane, except $\omega R \rightarrow \omega R / 2$. So, the outer region of the M2-brane is mapped into the inner region of the M5-brane and vice versa. The tunneling 
probability between the two regions must be the same function as for the M5 brane. So, here we find

$$
P=\frac{\pi}{9}(\omega R / 2)^{9}
$$

which agrees with the direct calculation [33].

Now, let us look at the metric. The M2-brane metric is

$$
\begin{gathered}
d s^{2}=A(r)^{-2 / 3} d x_{\|}^{2}+A(r)^{1 / 3}\left(d r^{2}+r^{2} d \Omega_{7}^{2}\right), \\
A(r)=1+\frac{R^{6}}{r^{6}}
\end{gathered}
$$

Defining the coordinate $z=R^{3} /\left(2 r^{2}\right)$, we find

$$
d s^{2}=\frac{R^{2}}{4 z^{2}}\left[B(z)^{-2 / 3} d x_{\|}^{2}+B(z)^{1 / 3}\left(d z^{2}+4 z^{2} d \Omega_{7}^{2}\right)\right]
$$

where

$$
B(z)=1+\frac{R^{3}}{8 z^{3}}
$$

After a conformal transformation this becomes

$$
d s_{\text {new }}^{2}=B(z)^{-1 / 3} d x_{\|}^{2}+B(z)^{2 / 3}\left(d z^{2}+4 z^{2} d \Omega_{7}^{2}\right) .
$$

The $r t$ part of this metric coincides with that of the M5-brane.

Now we proceed to calculating corrections to the low energy absorption cross-sections.

\subsection{The D3-brane case}

The equation of motion for a minimal scalar $\phi$ in the $s$-wave is $(1)$. In terms of $y=(\omega R)^{2} / \rho$ and $\phi=y^{4} \psi$, we have

$$
\left[\frac{1}{y^{5}} \partial_{y} y^{5} \partial_{y}+1+\frac{(\omega R)^{4}}{y^{4}}\right] \psi=0 .
$$

The strategy we will employ is to expand

$$
\phi(\rho)=\phi_{0}(\rho)+(\omega R)^{4} \phi_{1}(\rho)+\ldots
$$

in the outer region where the last term in (1) is small. Writing down (1) order by order in $(\omega R)^{4}$, we find

$$
\begin{aligned}
& {\left[\frac{1}{\rho^{5}} \partial_{\rho} \rho^{5} \partial_{\rho}+1\right] \phi_{0}=0} \\
& {\left[\frac{1}{\rho^{5}} \partial_{\rho} \rho^{5} \partial_{\rho}+1\right] \phi_{1}=-\frac{1}{\rho^{4}} \phi_{0} .}
\end{aligned}
$$

The two solutions to the homogeneous equation for $\phi_{0}$ are

$$
\begin{aligned}
& \frac{J_{2}(\rho)}{\rho^{2}}=\frac{1}{8}\left(1-\frac{\rho^{2}}{12}+O\left(\rho^{4}\right)\right) \\
& \frac{N_{2}(\rho)}{\rho^{2}}=-\frac{4}{\pi \rho^{4}}\left(1+\frac{\rho^{2}}{4}+O\left(\rho^{4} \log \rho\right)\right) .
\end{aligned}
$$


Using a trick of second order differential equations, we can write down a solution of the inhomogeneous equation directly:

$$
\phi_{1}(\rho)=-\frac{\pi}{2 \rho^{2}} \int^{\rho} \frac{d \sigma}{\sigma} \phi_{0}(\sigma)\left(J_{2}(\sigma) N_{2}(\rho)-N_{2}(\sigma) J_{2}(\rho)\right)
$$

A convenient notation is to refer to $\phi_{1}$ as $\Delta \phi_{0}$. Note however that $\phi_{1}$ is ambiguous because one can add to it any solution to the homogeneous equation. This ambiguity is fixed by imposing the boundary conditions that 1) all flux at the horizon is infalling, and 2) the solution of inner and outer region match to order $(\omega R)^{4}$ in the transition region. ${ }^{5}$

The corrected solutions for the inner region $\mathbf{I}$ and outer region III are as follows:

$$
\begin{aligned}
\phi^{\mathbf{I}} & =y^{4}\left(\psi_{0}+(\omega R)^{4} \psi_{1}\right) \\
& =y^{2} H_{2}^{(1)}(y)+(\omega R)^{4} \frac{\pi y^{2}}{2} \int^{y} \frac{d x}{x^{3}} H_{2}^{(1)}(x)\left(J_{2}(x) N_{2}(y)-N_{2}(x) J_{2}(y)\right) \\
\frac{\phi^{\mathbf{I I I}}}{A} & =\phi_{0}+(\omega R)^{4} \phi_{1} \\
& =\frac{J_{2}(\rho)}{\rho^{2}}-(\omega R)^{4} \frac{\pi}{2 \rho^{2}}\left[\int^{\rho} \frac{d \sigma}{\sigma^{3}} J_{2}(\sigma)^{2} N_{2}(\rho)-\int^{\rho} \frac{d \sigma}{\sigma^{3}} J_{2}(\sigma) N_{2}(\sigma) J_{2}(\rho)\right] .
\end{aligned}
$$

By adjusting the contribution from the homogeneous terms of order $(\omega R)^{4}$, the wave function in the overlapping region can be made to match up to order $(\omega R)^{4} \log (\omega R)$

$$
\begin{aligned}
\phi^{\mathbf{I}} & =-\frac{4 i}{\pi}\left[1+\frac{y^{2}}{4}-\frac{(\omega R)^{4}}{12 y^{2}}\left(1-\frac{y^{2} \log y}{2}\right)\right]+\ldots \\
& =-\frac{4 i}{\pi}\left[1+(\omega R)^{2}\left(\frac{1}{4 z^{2}}-\frac{z^{2}}{12}\right)+(\omega R)^{4}\left(-\frac{1}{24} \log z+\frac{1}{24} \log \omega R\right)+\ldots\right] \\
\frac{\phi^{\mathbf{I I I}}}{A} & =\frac{1}{8}\left(1-\frac{\rho^{2}}{12}\right)+\frac{(\omega R)^{4}}{32 \rho^{2}}\left(1-\frac{1}{6} \rho^{2} \log \rho\right)+\ldots \\
& =\frac{1}{8}\left[1+(\omega R)^{2}\left(\frac{1}{4 z^{2}}-\frac{z^{2}}{12}\right)+(\omega R)^{4}\left(-\frac{1}{24} \log z-\frac{1}{24} \log \omega R\right)+\ldots\right]
\end{aligned}
$$

where we have introduced a new radial variable $z$ :

$$
z=r / R \quad \rho=(\omega R) z \quad y=\frac{\omega R}{z} .
$$

The self-dual point is $z=1$. The idea is to keep $z$ finite in the matching region, so that both $\rho$ and $y$ are small. Indeed, we observe a leading mismatch at order $(\omega R)^{4} \log (\omega R)$. If in $(32)$ we now choose

$$
A=-\frac{32 i}{\pi}\left[1+\frac{(\omega R)^{4}}{12} \log \omega R-O\left((\omega R)^{4}\right)\right]
$$

\footnotetext{
${ }^{5}$ As we will see below, the analysis of matching at the transition region will reveal a dominant correction of order $(\omega R)^{4} \log (\omega R)$ in the flux ratio, and if we are only interested in computing the absorption cross section to this order and not to order $(\omega R)^{4}$, we will be justified in ignoring the effect of homogeneous terms of order $(\omega R)^{4}$ on the asymptotic flux.
} 
then $\phi^{\mathbf{I}}$ and $\phi^{\mathbf{I I I}}$ match perfectly. The cross-section goes as $1 /|A|^{2}$, so the corrected result is

$$
\sigma=\frac{\pi^{4}}{8} \omega^{3} R^{8}\left(1-\frac{(\omega R)^{4}}{6} \log \omega R+O\left((\omega R)^{4}\right)\right) .
$$

We have verified the coefficient on the logarithm by numerically solving the radial equation (1). Note however that one has to get to very small $\omega R$ to see the logarithm numerically.

\subsection{The M5-brane case}

The methods for this case are the same as for the D3-brane, except in that it is necessary to iterate the outer region perturbation twice. The radial equation for a minimal scalar in the $s$-wave is (24). Defining

$$
y=\frac{2(\omega R)^{3 / 2}}{\sqrt{\rho}} \quad \phi=y^{3} \psi
$$

we also have

$$
\left[\frac{1}{y^{7}} \partial_{y} y^{7} \partial_{y}+1+\frac{64(\omega R)^{6}}{y^{6}}\right] \psi=0 .
$$

On can make the expansions

$$
\begin{aligned}
\phi & =\phi_{0}+(\omega R)^{3} \phi_{1}+(\omega R)^{6} \phi_{2}+\ldots \\
\psi & =\psi_{0}+(2 \omega R)^{6} \psi_{1}+\ldots
\end{aligned}
$$

It is necessary to calculate only the terms shown explicitly in (38):

$$
\begin{aligned}
\phi_{0} & =\frac{J_{3 / 2}(\rho)}{\rho^{3 / 2}}=\frac{1}{3} \sqrt{\frac{2}{\pi}}\left(1-\frac{\rho^{2}}{10}+\ldots\right) \\
\phi_{0} & =-\frac{\pi}{2 \rho^{3 / 2}}\left[\int^{\rho} \frac{d \sigma}{\sqrt{\sigma}} \phi_{0}(\sigma) J_{3 / 2}(\sigma) N_{3 / 2}(\rho)-\int^{\rho} \frac{d \sigma}{\sqrt{\sigma}} \phi_{0}(\sigma) N_{3 / 2}(\sigma) J_{3 / 2}(\rho)\right] \\
& =\frac{1}{3 \sqrt{2 \pi} \rho}\left(1-\frac{1}{5} \rho^{2}+\ldots\right) \\
\phi_{2} & =-\frac{\pi}{2 \rho^{3 / 2}}\left[\int^{\rho} \frac{d \sigma}{\sqrt{\sigma}} \phi_{1}(\sigma) J_{3 / 2}(\sigma) N_{3 / 2}(\rho)-\int^{\rho} \frac{d \sigma}{\sqrt{\sigma}} \phi_{1}(\sigma) N_{3 / 2}(\sigma) J_{3 / 2}(\rho)\right] \\
& =\frac{1}{6 \sqrt{2 \pi} \rho^{2}}\left(1-\frac{1}{5} \rho^{2} \log \rho+\ldots\right) \\
\psi_{0} & =\frac{H_{3}^{(1)}(y)}{y^{3}}=-\frac{16 i}{\pi y^{6}}\left(1+\frac{y^{2}}{8}+\frac{y^{4}}{64}+\ldots\right) \\
\psi_{1} & =-\frac{\pi}{2 y^{3}} \int^{y} \frac{d x}{x^{2}} \psi_{0}(x)\left(J_{3}(x) N_{3}(y)-N_{3}(x) J_{3}(y)\right) \\
& =\frac{2 i}{5 \pi y^{10}}\left(1+\frac{y^{2}}{4}-\frac{y^{4} \log y}{64}+\ldots\right) .
\end{aligned}
$$

In fact $\phi_{0}$ and $\phi_{1}$ can be given in closed form in terms of trigonometric functions. 
Now we can do the matching. Define a radial variable $z$ which is finite in the matching region:

$$
z=r / R, \quad \rho=\omega R z, \quad y=\frac{2 \omega R}{\sqrt{z}} .
$$

We obtain

$$
\begin{aligned}
\phi_{\mathbf{I}} & =y^{6}\left(\psi_{0}+(2 \omega R)^{6} \psi_{1}+\ldots\right) \\
& =-\frac{16 i}{\pi}\left[1+(\omega R)^{2}\left(\frac{1}{2 z}-\frac{z^{2}}{10}\right)+(\omega R)^{4}\left(\frac{1}{4 z^{2}}-\frac{z}{10}\right)+\frac{1}{10}(\omega R)^{6} \log \omega R+\ldots\right] \\
\frac{\phi_{\mathbf{I I I}}}{A} & =\phi_{0}+(\omega R)^{3} \phi_{1}+(\omega R)^{6} \phi_{2}+\ldots \\
& =\frac{1}{3} \sqrt{\frac{2}{\pi}}\left[1+(\omega R)^{2}\left(\frac{1}{2 z}-\frac{z^{2}}{10}\right)+(\omega R)^{4}\left(\frac{1}{4 z^{2}}-\frac{z}{10}\right)-\frac{1}{20}(\omega R)^{6} \log \omega R+\ldots\right] .
\end{aligned}
$$

A match is achieved to the order shown by setting

$$
A=-24 i \sqrt{\frac{2}{\pi}}\left(1+\frac{3}{20}(\omega R)^{6} \log \omega R\right),
$$

which means that the absorption probability is

$$
P=\frac{\pi}{9}(\omega R)^{9}\left(1-\frac{3}{10}(\omega R)^{6} \log \omega R+\ldots\right) .
$$

The coefficient on the logarithm matches the numerical analysis we have performed. To get the corresponding result for the M2-brane, we only need to replace $\omega$ by $\omega / 2$ in (43).

\section{The world-volume approach}

The world-volume analysis, as usual, is much simpler than the supergravity once one realizes what the relevant effects are. We will begin by considering a single D3-brane, where the action involves only an abelian gauge field and its superpartners. In the past [2, 3], it has been sufficient to generalize the single brane calculation to multiple branes simply by multiplying cross-sections by a factor of $N^{2}$ to account for the $N^{2}$ massless gauge fields on a group of $N$ coincident branes. This unexpected simplification is due to supersymmetric nonrenormalization theorems that sometimes imply that the one-loop result is not renormalized [4]. All the lowest order diagrams are contained in the abelian theory, so we may think of the abelian calculation as the leading term in the $g_{Y M}^{2} N$ expansion of the non-abelian calculation. Even if the coefficient is a non-trivial function of $g_{Y M}^{2} N$, it may approach a smooth infinite coupling limit, similar to the factor of $3 / 4$ in the entropy calculation [1].

Here we are dealing with a more complicated situation than that encountered in earlier literature, because the operators responsible for the effects we have seen in earlier sections are dimension 8, and to obtain their precise form one requires input from the non-abelian DBI action literature $[17,18,19]$. 
For processes that are on-shell in the world-volume theory, the only relevant coupling of the dilaton to the brane is through the gauge field, as was first realized in [2]. The action is

$$
\begin{aligned}
S_{\mathrm{DBI}} & =-T_{3} \int d^{4} x \sqrt{-\operatorname{det}\left(g_{\mu \nu}+\frac{e^{-\phi / 2}}{\sqrt{T_{3}}} F_{\mu \nu}\right)} \\
& =\int d^{4} x \sqrt{g}\left[T_{3}-\frac{1}{4} e^{-\phi} F^{2}-\frac{1}{8} \frac{e^{-2 \phi}}{T_{3}}\left(F^{4}-\frac{1}{4}\left(F^{2}\right)^{2}\right)+\ldots\right]
\end{aligned}
$$

where $g_{\mu \nu}$ is the induced Einstein metric on the brane, and we have renormalized $F_{\mu \nu}$ to give the photon propagator a pole of residue one. We have introduced a short-hand for Lorentz traces of $F_{\mu \nu}$ :

$$
F^{n}={F_{\mu_{1}}}^{\mu_{2}}{F_{\mu_{2}}}^{\mu_{3}} \ldots{F_{\mu_{n}}}^{{ }^{\mu_{1}}} .
$$

To make expressions compact it is useful to define

$$
\mathcal{O}_{4}=F^{2}, \quad \mathcal{O}_{8}=F^{4}-\frac{1}{4}\left(F^{2}\right)^{2} .
$$

From (44) one can obtain an expression for the trace of the stress-tensor:

$$
T_{\mu}^{\mu}=-\frac{1}{2} \frac{e^{-2 \phi}}{T_{3}} \mathcal{O}_{8}
$$

This operator is dimension 8 , suitable to match the scaling of the fixed scalar cross-section with energy.

At linear order, the dilaton couples to the world-volume through

$$
S_{\text {int }}=\int d^{4} x \phi \mathcal{O}_{\phi}=\int d^{4} x\left(-\frac{1}{4}\right) \phi\left[\mathcal{O}_{4}+\frac{1}{T_{3}} \mathcal{O}_{8}+\ldots\right] .
$$

From the two-point function of the renormalizable part of $\mathcal{O}_{\phi}$ one can read off the low-energy cross-section $\sigma=\frac{\kappa^{2} \omega^{3}}{32 \pi}$, which is correct even in normalization. Our present purpose is to consider the leading corrections to this two-point function. First of all, it is evident that in the expansion by operator dimension, the higher one goes the more inverse powers of $T_{3}$ show up in the correlators. Extra powers of momenta (or just energy in the case of normal incidence absorption cross-sections) appear as required by dimensional analysis. We are looking only for the first correction, with a single inverse power of $T_{3}$ and four powers of $\omega$, corresponding to $(\omega R)^{4}$ in supergravity. To the order which we desire, then,

$$
\begin{aligned}
\left\langle\mathcal{O}_{\phi}(x) \mathcal{O}_{\phi}(0)\right\rangle_{\mathrm{DBI}} & =\int \mathcal{D} A_{\mu} e^{-\int d^{4} y\left[\frac{1}{4} \mathcal{O}_{4}+\frac{1}{8 T_{3}} \mathcal{O}_{8}\right]} \mathcal{O}_{\phi}(x) \mathcal{O}_{\phi}(0) \\
& =\int \mathcal{D} A_{\mu} e^{-\int d^{4} y \frac{1}{4} \mathcal{O}_{4}} \mathcal{O}_{\phi}(x) \mathcal{O}_{\phi}(0)\left(1-\frac{1}{8 T_{3}} \int d^{4} z \mathcal{O}_{8}(z)\right) \\
& =\left\langle\mathcal{O}_{\phi}(x) \mathcal{O}_{\phi}(0)\left(1-\frac{1}{8 T_{3}} \int d^{4} z \mathcal{O}_{8}(z)\right)\right\rangle_{\mathrm{CFT}} \\
& =\frac{1}{16}\left[\left\langle\mathcal{O}_{4}(x) \mathcal{O}_{4}(0)\right\rangle_{\mathrm{CFT}}-\frac{1}{8 T_{3}} \int d^{4} z\left\langle\mathcal{O}_{4}(x) \mathcal{O}_{8}(z) \mathcal{O}_{4}(0)\right\rangle_{\mathrm{CFT}}\right]
\end{aligned}
$$




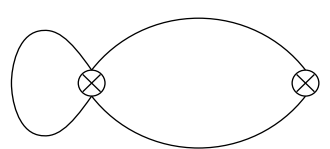

a)

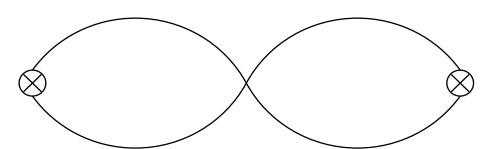

b)

Figure 1: The circled crosses $(\otimes)$ denote an insertion of $\mathcal{O}_{\phi}$.

The subscripted CFT on the correlators in the last two lines of (49) reminds us that these correlators are evaluated in the free gauge theory, which of course is conformal. From here on we will drop this subscript: all correlators will be evaluated in the conformal theory. Note that we have gone to Euclidean signature in order to simplify calculations. In the last line of (49) we have suppressed terms like $\left\langle\mathcal{O}_{4}(x) \mathcal{O}_{8}(0)\right\rangle$ coming from the dimension eight corrections to the vertex operator (48), which in principle seem to contribute at the same order. From a conformal field theory point of view, these terms should vanish because only operators of the same dimension can have a non-vanishing two point function. From a diagrammatic point of view, one can arrange for them to vanish by assuming (as we will below) that $\mathcal{O}_{4}$ and $\mathcal{O}_{8}$ are normal ordered. Even if they were not, their contributions would amount only to (divergent) contributions to the absorption probability of order $(\omega R)^{4}$ without a log. In other words, a full analysis of the pure $(\omega R)^{4}$ correction might require us to consider diagrams like those in figure 1a; but the leading log correction comes only from the figure eight diagrams shown in figure $1 b$.

Now let us proceed to explicit evaluation of the free field correlators. The relevant part of the action is

$$
\frac{1}{4}(1-\phi) F^{2}+\frac{1-2 \phi}{8 T_{3}}\left(F^{4}-\frac{1}{4}\left(F^{2}\right)^{2}\right)
$$

In Euclidean space, and in Feynman gauge, the propagator is

$$
\left\langle A_{\mu}(x) A_{\nu}(0)\right\rangle=\frac{\delta_{\mu \nu}}{4 \pi^{2} x^{2}}
$$

from which one can infer the (gauge-invariant) propagator for the field strength

$$
\left\langle F_{\mu \nu}(x) F_{\alpha \beta}(0)\right\rangle=\frac{1}{2 \pi^{2} x^{4}}\left(\delta_{[\alpha}^{[\beta} \delta_{\mu]}^{\nu]}-\frac{4}{x^{2}} \delta_{[\alpha}^{[\beta} x_{\mu]} x^{\nu]}\right)
$$

Because we have rotated to Euclidean signature, there is no difference between upper and lower indices. Using standard perturbative methods, one finds

$$
\Pi_{0}(x)=\left\langle: \frac{1}{4} F^{2}(x):: \frac{1}{4} F^{2}(0):\right\rangle=\frac{3}{\pi^{4}} \frac{1}{x^{4}}
$$

which gives the leading contribution, and

$$
\Pi_{1}(x)=-\int d^{4} z\left\langle: \frac{1}{4} F^{2}(x):: \frac{1}{8 T_{3}}\left(F^{4}-\frac{1}{4}\left(F^{2}\right)^{2}\right)(z):: \frac{1}{4} F^{2}(0):\right\rangle=-\int d^{4} z \frac{9}{\pi^{8} T_{3}} \frac{1}{z^{8}(x-z)^{8}}
$$


which gives the correction arising from the "figure 8" diagram of figure 1b. This form follows from operator product expansions

$$
\mathcal{O}_{4}(z) \mathcal{O}_{4}(0)=\frac{1}{z^{8}} \mathbf{1}+\mathcal{O}_{8}(0)+\ldots, \quad \mathcal{O}_{8}(z) \mathcal{O}_{4}(0)=\frac{1}{z^{8}} \mathcal{O}_{4}(0)+\ldots
$$

In momentum space, they become

$$
\begin{aligned}
& \Pi_{0}(p)=\int d^{4} x \Pi_{0}(x) e^{i p x}=\frac{p^{4}}{64 \pi^{2}} \log \left(p^{2} / \Lambda^{2}\right) \\
& \Pi_{1}(p)=\int d^{4} x \Pi_{1}(x) e^{i p x}=-\frac{p^{8}}{4096 \pi^{4} T_{3}}\left(\log \left(p^{2} / \Lambda^{2}\right)\right)^{2}
\end{aligned}
$$

As was explained in [4], absorption cross section can be inferred from the discontinuity across the positive real axis in the $s$-plane $\left(s=-p^{2}\right)$ of $\Pi_{0}$ and $\Pi_{1}$.

$$
\sigma=\left.\frac{2 \kappa^{2}}{2 i \omega} \operatorname{Disc}\left(\Pi_{0}(s)+\Pi_{1}(s)\right)\right|_{\substack{p^{0}=\omega \\ \vec{p}=0}}=\frac{\kappa^{2} \omega^{3}}{32 \pi}\left(1-\frac{1}{8}(\omega R)^{4} \log (\omega / \Lambda)\right)
$$

where we used the relation between radius of the throat $R$ and the 3 -brane tension $T_{3}$,

$$
R^{4}=\frac{N}{2 T_{3} \pi^{2}}=4 \pi g N \alpha^{\prime 2}
$$

This is precisely the form of the dependence on $\omega$ we found in the previous section using supergravity methods. The coefficient of the $\log (\omega)$ term disagrees from the supergravity calculation by a factor of $4 / 3$. This is not necessarily a contradiction, however. One must keep in mind that we trust the supergravity calculation in the limit where $g_{Y M}^{2} N$ is large. From the world volume point of view, one must take proper account of the non-abelian nature of the theory. This might lead to a non-trivial dependence on $g_{Y M}^{2} N$ which approaches a smooth limit as $g_{Y M}^{2} N$ is sent to infinity, just as in the case of the entropy calculation [1].

In the full non-abelian theory the calculation of the correction to the absorption crosssection once again reduces to the correlator $\left\langle\mathcal{O}_{4}(x) \mathcal{O}_{8}(z) \mathcal{O}_{4}(0)\right\rangle$, except now we use the non-abelian operators given in (4) and (7). Due to the constraints of conformal invariance, the 3-point function is, up to an overall function of $g_{Y M}^{2} N$, the same as at the leading order in $g_{Y M}^{2} N$,

$$
\left\langle\mathcal{O}_{4}(x) \mathcal{O}_{8}(z) \mathcal{O}_{4}(0)\right\rangle \sim \frac{1}{z^{8}(x-z)^{8}} .
$$

According to the discussion above, this guarantees that the absorption cross-section has the form

$$
\frac{\kappa^{2} \omega^{3}}{32 \pi}\left(1-f\left(g_{Y M}^{2} N\right) \omega^{4} N \kappa \log (\omega / \Lambda)\right) .
$$

However, conformal invariance alone does not fix the function $f\left(g_{Y M}^{2} N\right)$. Our perturbative calculation tells us that $f(0)=1 / 8$. On the other hand, since the supergravity calculation is reliable in the limit of large $g_{Y M}^{2} N$, we can use (35) to deduce that $f=1 / 6$ in this limit. This in turn fixes the normalization of the 3-point function in (54). Of course, a detailed understanding of the strong coupling limit of the world-brane theory will be required to check it. 
A comment is in order regarding the ultraviolet cut-off scale $\Lambda$. In order to match the supergravity result (35), one should set $\Lambda=1 / R$. On the other hand, these world volume theories are an effective description of open strings ending on a D-brane, and the natural ultraviolet cut-off is $\Lambda=1 / \sqrt{\alpha^{\prime}}$. However, for arbitrary choice of the cut-off scale $\Lambda$, supergravity and world volume calculations differ only by a quantity of order

$$
(\omega R)^{4} \log (\omega R)-(\omega R)^{4} \log (\omega / \Lambda)=(\omega R)^{4} \log (\Lambda R)
$$

which can be thought of as being part of the $(\omega R)^{4}$ term (without the logarithm) which is subleading. Proper understanding of the ultraviolet cutoff therefore requires extending our analysis to this order. It would be interesting to carry out this analysis, perhaps by looking at the string theory amplitudes. We leave this point for future investigations.

A similar story appears to hold for the M2 and M5 branes. For the M5-brane, minimal and fixed scalars couple to operators of dimensions 6 and 12, respectively. Assuming that the the operator product expansion are of the form

$$
\mathcal{O}_{6}(z) \mathcal{O}_{6}(0)=\frac{1}{z^{12}} \mathbf{1}+\mathcal{O}_{12}(0)+\ldots, \quad \mathcal{O}_{12}(z) \mathcal{O}_{6}(0)=\frac{1}{z^{12}} \mathcal{O}_{6}(0)+\ldots
$$

conformal invariance will fix their correlation functions to take the form

$$
\Pi_{0}(x)=\frac{1}{x^{12}}, \quad \Pi_{1}(x)=\int d^{6} z \frac{1}{z^{12}(x-z)^{12}}
$$

On the $s$-space, they become

$$
\Pi_{0}(s)=s^{3} \log (-s), \quad \Pi_{1}(s)=s^{6} \log (-s)^{2}
$$

whose discontinuity on the real axis is

$$
\operatorname{Disc}\left(\Pi_{0}(s)+\Pi_{1}(s)\right)=i s^{3}\left(1+s^{3} \log (s)\right)
$$

which gives the desired $(\omega R)^{6} \log (\omega / \Lambda)$ dependence of the sub-leading term.

The pattern that appears to be emerging is that when minimal scalars couple to the operator on the brane world volume of dimension $d$, there is a leading logarithmic correction at order $(\omega R)^{d} \log (\omega / \Lambda)$. On the M2-brane, minimal scalars couple to operators of dimension 3. This appears to suggest that there is a logarithmic correction of order $(\omega R)^{3} \log (\omega / \Lambda)$ which would contradict our earlier result from supergravity calculation, where the leading correction to the minimal scalar absorption was found to be of order $(\omega R)^{6} \log (\omega / \Lambda)$. Quite happily, we find that the conformal field theory on M2 knows about the absence of $(\omega R)^{3} \log (\omega / \Lambda)$ correction upon closer examination. Let us elaborate on this point.

The dimension of operators on M2 world volume coupling to the minimal and fixed scalars are 3 and 6, respectively. Assuming that the the operator product expansion are again of the form

$$
\mathcal{O}_{3}(z) \mathcal{O}_{3}(0)=\frac{1}{z^{6}} \mathbf{1}+\mathcal{O}_{6}(0)+\ldots, \quad \mathcal{O}_{6}(z) \mathcal{O}_{3}(0)=\frac{1}{z^{6}} \mathcal{O}_{3}(0)+\ldots
$$

conformal invariance will fix their correlation functions to take the form

$$
\Pi_{0}(x)=\frac{1}{x^{6}}, \quad \Pi_{1}(x)=\int d^{3} z \frac{1}{z^{6}(x-z)^{6}}
$$



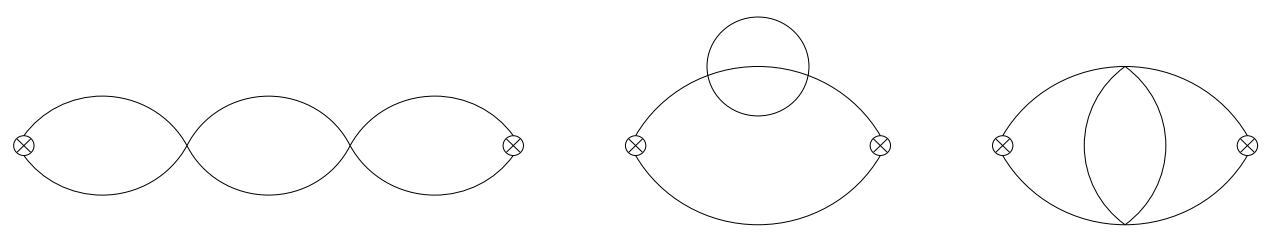

Figure 2: The three loop contributions to the two point function.

This time, we find that on $s$-space, they become

$$
\Pi_{0}(s)=(-s)^{3 / 2}, \quad \Pi_{1}(s)=s^{3} .
$$

The leading non-analytic behavior is a square root instead of the logarithm. In particular, $\Pi_{1}(s)$ has no non-analyticity what so ever, and we find

$$
\operatorname{Disc}\left(\Pi_{0}(s)+\Pi_{1}(s)\right)=i s^{3 / 2}
$$

which gives the desired leading term, and the desired absence of $(\omega R)^{3} \log (\omega / \Lambda)$ correction. Power counting in $\omega$ and $R$ indicates that the desired $(\omega R)^{6} \log (\omega / \Lambda)$ correction should arise from the next correction to the two point function:

$$
\Pi_{2}(x)=\int d^{3} z_{1} d^{3} z_{2}\left\langle\mathcal{O}_{3}(x) \mathcal{O}_{6}\left(z_{1}\right) \mathcal{O}_{6}\left(z_{2}\right) \mathcal{O}_{3}(0)\right\rangle
$$

To see what singularities arise from (56) it is useful to replace the interacting CFT of the M2 world-volume with a free CFT: for example a free conformally coupled scalar $\phi$. Then, roughly speaking, $\mathcal{O}_{3}$ could be realized as $(\partial \phi)^{2}$ and $\mathcal{O}_{6}$ as $(\partial \phi)^{4}$. The three diagrams arising from the Wick contractions of (56) are shown in figure 2. The first two only contribute $s^{9 / 2}$ corrections to (55); the last however contains overlapping divergences and can contribute a $s^{9 / 2} \log (s)$ term to $(55)$ through the Fourier transform of integrals like

$$
\int d^{3} z_{1} d^{3} z_{2} \frac{1}{\left(x-z_{1}\right)^{3}} \frac{1}{\left(x-z_{2}\right)^{3}} \frac{1}{z_{1}^{3}} \frac{1}{z_{2}^{3}} \frac{1}{\left(z_{1}-z_{2}\right)^{6}} \sim \frac{\log \Lambda x}{x^{12}} .
$$

A coupling of the minimal scalar to $\mathcal{O}_{6}$ does not seem to give additional logarithmic contributions.

\section{Conclusions}

The proposal [6] that the world-volume theory of a large number of coincident branes should be identified with string theory in the background of the near-horizon geometry is an elaboration of earlier work $[2,3,4]$ where the entire brane geometry (which goes beyond the throat approximation) was used. In this paper we studied some of the effects that go beyond the throat approximation and argued that they are just as universal as the strict conformal limit.

We have presented two closely related calculations. First, we identified a non-minimally coupled "fixed" scalar and showed that a coupling of this scalar to the trace of the stressenergy tensor of the full DBI action reproduces the supergravity cross-section up to a normalization. We regard the normalization question in this case as something of a fine point: 
as in comparisons of matrix models to Liouville theory, the things to compare between the world-volume theory and supergravity are ratios of two and three point functions which are independent of normalizations.

The calculation of logarithmic corrections to the energy dependence of the minimal scalar cross-section strikes more deeply at the physics of D-branes: whereas the world-volume calculation for fixed scalar required the DBI action only in order to find the correct vertex operator for the fixed scalar, the logarithmic corrections to minimal scalar cross-sections arise from considering DBI corrections to the conformal theory in the path integral.

One striking feature of our results is that the semi-classical supergravity is being compared with the $\alpha^{\prime}$ corrections to the DBI action, always thought to be a purely string theoretic effect. Thus, we are finding out that the supergravity 'knows' string theory.

Our calculations suggest an extension of the throat-brane correspondence conjecture, which was implicit in the earlier work of $[2,3,4]$ : the world-volume theory of $N$ coincident D3-branes interacting with closed strings in the bulk is equivalent to type IIB string theory in the background of the self-dual black threebrane carrying $N$ units of RR charge. One can make a more concrete claim at low energy: expanding departures in the world-volume theory from conformal invariance in a series of irrelevant perturbations to the Yang-Mills action corresponds to considering supergravity in the throat region, but with the usual $A d S$ throat metric replaced by the true D3-brane metric. In this paper we compared the leading correction to the absorption cross-section in the double-scaling limit (2) proposed in [2]. ${ }^{6}$ Our calculation of the corrections due to the first irrelevant perturbation, together with the work on BIons in $[34,35,36,37]$, perhaps constitute only the beginning of a fuller understanding of the relation between the non-abelian DBI action and string theory in the brane background.

\section{Acknowledgement}

We would like to thank A. Tseytlin and the participants of the String Dualities program at ITP, Santa Barbara, for discussions. S.S.G. and I.R.K. are grateful to ITP for hospitality during the completion of this paper. This research was supported in part by the National Science Foundation under Grant No. PHY94-07194, by the Department of Energy under Grant No. DE-FG02-91ER40671, and by the James S. McDonnell Foundation under Grant No. 91-48. S.S.G. also thanks the Hertz Foundation for its support.

\section{References}

[1] S. S. Gubser, I. R. Klebanov, and A. W. Peet, "Entropy and temperature of black 3-branes," Phys. Rev. D54 (1996) 3915-3919, hep-th/9602135.

[2] I. R. Klebanov, "World volume approach to absorption by nondilatonic branes," Nucl. Phys. B496 (1997) 231, hep-th/9702076.

\footnotetext{
${ }^{6}$ It would be very interesting to see if the higher order corrections can be compared explicitly. In fact, we conjecture that the semi-classical absorption cross-section calculated as a function of $(\omega R)^{4}$ from the wave equation (1) defines a "universal function" which should be reproduced by the non-abelian DBI theory in the double-scaling limit (2).
} 
[3] S. S. Gubser, I. R. Klebanov, and A. A. Tseytlin, "String theory and classical absorption by three-branes," Nucl. Phys. B499 (1997) 217, hep-th/9703040.

[4] S. S. Gubser and I. R. Klebanov, "Absorption by branes and Schwinger terms in the world volume theory," hep-th/9708005.

[5] A. M. Polyakov, "String theory and quark confinement," hep-th/9711002.

[6] J. Maldacena, "The Large N limit of superconformal field theories and supergravity," hep-th/9711200.

[7] S. S. Gubser, I. R. Klebanov, and A. M. Polyakov, "Gauge theory correlators from noncritical string theory," hep-th/9802109.

[8] G. T. Horowitz and H. Ooguri, "Spectrum of large N gauge theory from supergravity," hep-th/9802116.

[9] E. Witten, "Anti-de Sitter space and holography," hep-th/9802150.

[10] S. Kachru and E. Silverstein, "4-D conformal theories and strings on orbifolds," hep-th/9802183.

[11] S. Ferrara, C. Fronsdal, and A. Zaffaroni, "On N=8 Supergravity on $A d S_{5}$ and $\mathrm{N}=4$ Superconformal Yang-Mills theory," hep-th/9802203.

[12] J. Maldacena, "Wilson loops in large $N$ field theories," hep-th/9803002.

[13] A. Lawrence, N. Nekrasov, and C. Vafa, "On Conformal Field Theories in Four Dimensions," hep-th/9803015.

[14] G. W. Gibbons and P. K. Townsend, "Vacuum interpolation in supergravity via super p-branes," Phys. Rev. Lett. 71 (1993) 3754-3757, hep-th/9307049.

[15] G. W. Gibbons, G. T. Horowitz, and P. K. Townsend, "Higher dimensional resolution of dilatonic black hole singularities," Class. Quant. Grav. 12 (1995) 297-318, hep-th/9410073.

[16] S. R. Das, "The Effectiveness of D-branes in the description of near extremal black holes," Phys. Rev. D56 (1997) 3582-3590, hep-th/9703146.

[17] A. A. Tseytlin, "Vector Field Effective Action in the Open Superstring Theory," Nucl. Phys. B276 (1986) 391.

[18] D. J. Gross and E. Witten, "Superstring Modifications of Einstein's Equations," Nucl. Phys. B277 (1986) 1.

[19] A. A. Tseytlin, "On non-Abelian generalization of Born-Infeld action in string theory," Nucl. Phys. B501 (1997) 41, hep-th/9701125.

[20] B. Kol and A. Rajaraman, "Fixed scalars and suppression of Hawking evaporation," Phys. Rev. D56 (1997) 983-986, hep-th/9608126. 
[21] C. G. Callan, S. S. Gubser, I. R. Klebanov, and A. A. Tseytlin, "Absorption of fixed scalars and the D-brane approach to black holes," Nucl. Phys. B489 (1997) 65-94, hep-th/9610172.

[22] I. R. Klebanov and M. Krasnitz, "Fixed scalar gray body factors in five-dimensions and four- dimensions," Phys. Rev. D55 (1997) 3250-3254, hep-th/9612051.

[23] M. Krasnitz and I. R. Klebanov, "Testing effective string models of black holes with fixed scalars," Phys. Rev. D56 (1997) 2173-2179, hep-th/9703216.

[24] H. W. Lee, Y. S. Myung, and J. Y. Kim, "Absorption of fixed scalars in scattering off 5-D black holes," hep-th/9708099.

[25] H. W. Lee, Y. S. Myung, and J. Y. Kim, "Absorption of fixed scalar in scattering off 4-D N=4 black holes," Phys. Lett. B410 (1997) 6, hep-th/9704199.

[26] S. Ferrara and R. Kallosh, "Supersymmetry and attractors," Phys. Rev. D54 (1996) 1514-1524, hep-th/9602136.

[27] S. Ferrara and R. Kallosh, "Universality of supersymmetric attractors," Phys. Rev. D54 (1996) 1525-1534, hep-th/9603090.

[28] S. Ferrara, R. Kallosh, and A. Strominger, "N=2 extremal black holes," Phys. Rev. D52 (1995) 5412-5416, hep-th/9508072.

[29] I. R. Klebanov, A. Rajaraman, and A. A. Tseytlin, "Intermediate scalars and the effective string model of black holes," Nucl. Phys. B503 (1997) 157, hep-th/9704112.

[30] G. T. Horowitz and A. Strominger, "Black strings and P-branes," Nucl. Phys. B360 (1991) 197-209.

[31] M. Dine and N. Seiberg, "Comments on higher derivative operators in some SUSY field theories," Phys. Lett. B409 (1997) 239-244, hep-th/9705057.

[32] H. J. Kim, L. J. Romans, and P. van Nieuwenhuizen, "The Mass Spectrum of Chiral N=2 d = 10 Supergravity on $S^{5}$," Phys. Rev. D32 (1985) 389.

[33] R. Emparan, "Absorption of scalars by extended objects," hep-th/9706204.

[34] C. G. Callan and J. M. Maldacena, "Brane dynamics from the Born-Infeld action," hep-th/9708147.

[35] G. W. Gibbons, "Born-Infeld particles and Dirichlet p-branes," hep-th/9709027.

[36] S. Lee, A. Peet, and L. Thorlacius, "Brane waves and strings," Nucl. Phys. B514 (1998) 161-176, hep-th/9710097.

[37] S.-J. Rey and J. Yee, "Macroscopic Strings as Heavy Quarks of Large N Gauge Theory and Anti-de Sitter Supergravity," hep-th/9803001. 